\title{
The Effect of Processing and Ingredient Interactions on Thiamine Degradation in Canned Cat Food: A Modern Nutrition-Health Dilemma
}

\author{
Molnar $\mathrm{L}^{2}$, Aldrich $\mathrm{CG}^{1}{ }^{*}$, and Trible $\mathrm{SD}^{3}$ \\ ${ }^{1}$ Research Associate Professor of Agriculture, Kansas State University, USA \\ ${ }^{2}$ Graduate research assistant, Kansas State University, USA \\ ${ }^{3}$ Senior Scientist, Purina Animal Nutrition LLC, USA
}

Review Article

Volume 2 Issue 2

Received Date: February 18, 2017

Published Date: March 27, 2017

*Corresponding author: Greg Aldrich, Research Associate Professor, Kansas State University, 201 Shellenberger Hall, 1301 Mid-Campus Dr, Manhattan KS, USA, 66506, E-mail: aldrich4@ksu.edu

\section{Abstract}

Water soluble vitamins play an integral role in normal metabolic function in cats. Thiamine deficiencies are a common issue in the pet food industry because thiamine degrades easily during processing. Specifically, when cats are fed a diet low in thiamine they may develop life threatening health issues including anorexia, ventroflexion, neurological impairment, and possibly death within a few weeks if not treated. However, little research has been published using a pet food matrix regarding what specific factors in pet food processing result in the most losses and whether these can be controlled. Thiamine can be degraded in a canned food due to heat, moisture, long-term storage, sulfites, $\mathrm{pH}$, and thiaminase enzyme activity. Thermal processes used to produce wet pet foods sold in cans, pouches, and trays are required to be heat treated for extended periods of time. This is detrimental to thiamine retention. Because the cat, like other carnivores, has a very high metabolic requirement for thiamine, they are susceptible to these losses. For this reason, supplementation is often a logical step. However, survival of more than $10 \%$ of the thiamine may not be assured. This review summarizes the prevailing literature on the topic with application to pet food. Further, suggestions regarding potential investigations to remedy the issue are discussed. Finding an optimal time $\mathrm{x}$ temperature $\mathrm{x} \mathrm{pH} \mathrm{x}$ ingredient combination is a real possibility that has the potential to save many cats in the future.

Keywords: Thiamine, Nutrition, Cat, Canning, Retort

\section{Thiamine and Pet Food}

Vitamins are essential nutrients for companion animal diets that must be provided in small amounts in order to perform biochemical and physiological functions. This is because essential vitamins are either not made by the animal or not produced in sufficient quantities to support vital needs. Thiamine, or Vitamin
B1, as it is often described, is one of these essential vitamins. Previous research has concluded that thiamine is susceptible to losses during canning. Jansen, et al. [1] reported that thiamine degrades due to heat processing, and alkaline conditions in canned meat products, and during storage. Since 2008, there have been numerous thiamine related recalls [2] affecting companies and products such as Iams Proactive Health Cat and Kitten Food (FDA, 2011), Nestle Well Pet LLC 
(FDA, 2011) Nestle Purina (FDA, 2012), and JM Smucker Company (FDA, 2017). This is not an issue of negligence [3]. Companies struggle to identify the proper level of supplementation for thiamine to compensate for the processing losses that can exceed $90 \%$ [4].

This is important because carnivores such as the domestic cat exhibit rather acute thiamine deficiency signs within a few weeks [5]. In their work Moon, et al. [6] fed male cats a cooked meat diet and female cat's stale food diets. The male cats exhibited signs of thiamine deficiency within two weeks and female cats three months. Clinical signs of thiamine deficiency present as anorexia, and changes in the central nervous system including seizures, head twitching, muscle weakness, incoordination, tachycardia, bradycardia, weight loss, and death [7-9]. Other common clinical signs of thiamine deficiency include ocular issues like acute blindness, gastrointestinal issues such as vomiting, weight loss, and constipation [10]. Ventroflexion, a flexion of the spine with movement of the head towards the chest, has been reported as a result of thiamine deficiency, and cats with the deficiency reported to somersault when they jumped from a table to the floor. It has also been reported that cats manifesting thiamine deficiency had more difficulty learning to complete tasks [11]. Thiamine deficiency also has been reported to cause an overproduction of pyruvate leading to ischemic cells in the brain [7]. The biochemical lesions damage an animal's carbohydrate metabolism and the animal can eventually die within four weeks if not treated with thiamine supplements [5]. Once a thiamine deficiency has been diagnosed, typically, a vitamin B complex injection is administered for three to five days, followed by oral dose for two to four weeks [12]. Thiamine deficiency is a health issue for cats and a potential economic problem for manufacturers of cat food because of its constant need by the animal and devastating result if left unaddressed.

Because thiamine is water soluble, it can only be stored in small limited amounts and is mostly lost through urine [13]. Therefore, nutrients such as thiamine must be provided and (or) supplemented in the food on a continuous basis. Cats are obligate carnivores that require high levels of protein and fatty acids [14]. They need thiamine in order to utilize enzymes in several metabolic pathways including the urea cycle to adjust to nitrogen levels in the body [14]. Cats also need thiamine for synthesis of fatty acids, amino acids, and carbohydrates [13]. If the diet is insufficient in thiamine a deficiency can develop into neurological issues and can result in death if supplementation is not provided to overcome the deficiency [15].
Thiamine, (2-\{3-[(4-amino-2-methylpyrimidin-5-yl) methyl]-4-methylthiazol-5-yl\} ethanol), like other water-soluble "B vitamins" are coenzymes that support nutrient utilization [16]. Water soluble B vitamins also aid in the production of metabolic energy through the TCA cycle [14-17]. Thiamine acts as a coenzyme known as pyrophosphate that becomes di-phosphorylated into thiamine diphosphate (TPP; Analytical Methods Committee, 1999). Once thiamine has been dephosphorylated, it receives electrons in an oxidationreduction process, and then catalyzes the conversion of pyruvate to acetyl-CoA [18]. Thiamine is important for carbohydrate metabolism, and plays a key role in brain development, function, maintenance, and communication processes $[19,20]$. In its cationic form, thiamine plays a role in the generation of acetylcholine, which stimulates cerebellum activity, and aids in nerve tissue repair [17].

Thiamine was the first water-soluble B vitamin to be discovered. Characterization began in 1890 with the recognition of beriberi disorder in humans related to the lack of an essential food element. This was later determined to be thiamine deficiency [21]. Thiamine was isolated in 1911, and found naturally in grain products such as rice and wheat germ, as well as yeast, legumes, and meat organs, particularly liver, heart, and kidney [3]. However, it was not correctly identified and published until 1936 [18]. Thiamine is produced naturally by organisms including plants, fungi, and bacteria [22]. There are several common pet food ingredients that contain thiamine in meaningful quantities and include yeast, peas, beans, wheat germ, oats, some meats (including pork and beef), and soy $[14,23]$. However, these ingredients either lack sufficient thiamine to fully support the diet or are limited in the diet due to other considerations. Thus, supplementation is generally required.

There are several phosphorylated forms of thiamine in the body including the active form, thiamine pyrophosphate. Thiamine Pyrophosphate is the coenzyme used in energy production [7]. Thiamine exists physiologically in various thiamine esters including thiamine monophosphate, thiamine diphosphate, and thiamine tri phosphate [24]. But, tissue based thiamine from meat and plant sources in the basal dietary ingredients are not typically included in commercial cat foods in an amount sufficient to support their needs. The two main supplemental forms of thiamine added to pet food are thiamine hydrochloride and thiamine mononitrate. Thiamine mononitrate is used more often [15]. Thiamine hydrochloride is a sulfur-containing vitamin composed of a thiazole ring and a pyrimidine group joined by a methylene (Figure 1) $[17,25,26]$. Thiamine is 


\section{Open Access Journal of Veterinary Science \& Research}

manufactured synthetically by oxidizing thiothiamine to thiamine sulfate in the presence of hydrogen peroxide and then salts are produced under ion-exchange $[27,28]$. Specifically, thiamine mononitrate is produced by oxidizing thiamine sulfate into an ethanol solution [28]. Then the final product is precipitated with several filters and dried under vacuum. Where thiamine hydrochloride is produced by oxidizing thiamine sulfate using ion exchange resin. Then the thiamine product is decolorized, distilled, precipitated, isolated through centrifugation, and dried under vacuum [28]. The structure of thiamine aids in the understanding of thiamine's instability through the canning process. The methylene bridge connecting the pyrimidine and thiazole moiety can easily be broken down by ingredients such as sulfites [29]. The thiazole moiety is less stable than the pyrimidine structure, and is easily cleaved by hydrolysis. The methylene bridge and sulfur ion within thiamine contribute to its instability during processing. Thiamine is susceptible to degradation during canning due to its ability to dissolve in water. Thiamine is most likely dissolved into water because of the bonding of the hydrogen atoms to the hydroxyl groups and amine tertiary structures, the near neutral $\mathrm{pH}$ in canned pet food products, and exposure to high heat for extended periods of time.

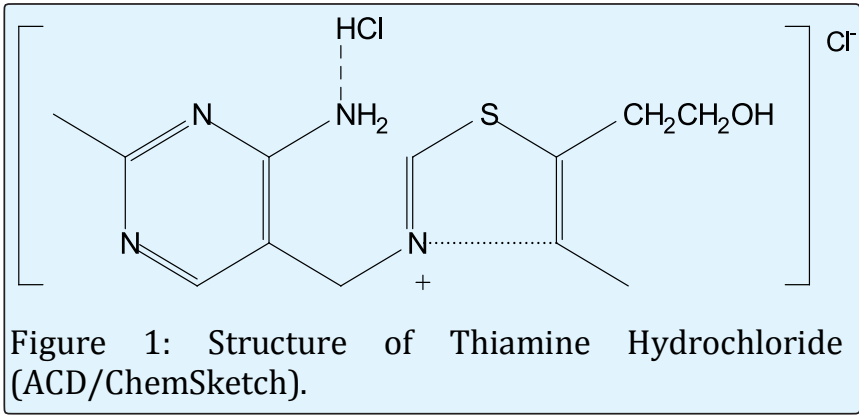

Thiamine requirements for cats and dogs were established by the National Research Council based on the limited amount of research in the target species [3]. Like most carnivores, cats have a relatively high thiamine requirement $(5.60 \mathrm{mg} / \mathrm{kg}$ diet $\mathrm{DM}$ on a 4,000 Kcal diet); whereas, dogs as more omnivorous species require a lower level $(1 \mathrm{mg} / \mathrm{kg}$ diet DM on a $4,000 \mathrm{Kcal}$ diet; [30]. Cats in late gestation have a higher thiamine requirement $(6.3 \mathrm{mg} / \mathrm{kg}$ diet DM on a $4,000 \mathrm{Kcal}$ diet). This difference in the amount of thiamine required, is in part, why thiamine deficiency in thermally processed canned foods may be more prevalent in foods produced for cats than dogs.

\section{Thermal Processing}

Thermal processing is critical to the food industry because it helps increase the safety of commercial products while transforming the food matrix into a convenient form for nutrient delivery to the animal [3135]. Canned food is produced through a cooking process (commercial sterilization) in which high temperature treatment is intended to destroy microorganisms and prevent growth of Clostridium botulinum spores [32,33]. "Commercial sterilization" is defined as thermal processing that will render the food free of microorganisms capable of reproducing in the food in normal non-refrigerated conditions during storage and distribution (21 CFR 113.3). Whereas full sterilization is defined as using a chemical or physical process that eliminates microorganisms [34]. Canned products are hermetically sealed to prevent unwanted growth of microorganisms with high heat resistance and it also prevents entry of pathogens that might produce toxins during storage [33].

For regulatory compliance (21CFR113) all canned foods must have a kinetic study on file reporting time vs temperature necessary to achieve bacterial lethality and verified by the process authority as validation that a product is commercially sterile $[33,36]$. The process authority will conduct a lethality studying measuring the F-values of canned products. The F-value measures the amount time at a constant temperature rate to kill a specific number of organisms such as $C$. botulinum using a $12 \log$ cycle reduction $[37,38]$.

A heat penetration study is completed to collect data for calculating commercial sterility [37]. Several factors that affect heat penetration of a canned product include retort temperature, processing time, initial temperature, size of container, shape of container, type of retort (still, agitating, etc), product formula, container type, and vacuum on the container [39]. The minimal Fvalue for pet food is 6 [37].

Even though thermal processing is required during the canning process, heat can degrade certain nutrients in the product and erode product quality. For example, Hendrik's, et al. [32], reported that digestibility of amino acids in a rat model decreased as heat processing time increased in an autoclave. This research group stated "to maintain optimal amino acid digestibility the amount of time for thermal processing should be limited. "This balance between safety and nutrient destruction could be true for vitamins like thiamine as well. 


\section{Open Access Journal of Veterinary Science \& Research}

\section{Processing Conditions and their Effect on Thiamine}

Within the parameters of the regulations there are several processing conditions to consider when exploring mechanisms involved with thiamine degradation in a canned pet food application. Ariahu, et al. [40] measured the retention of thiamine in brine, sauce, and soup formulated with periwinkle (Tympanostomusfuscatus) in a low acidic condition and processed at four different temperatures $(0$ to 40 minutes) and found that thiamine retention followed first order kinetics [40]. This means that the thiamine decay in the study was directly proportional to the time and temperature. This was confirmed by Durance et al. and Rehka et al. [36]. But does the degradation for thiamine occur simultaneous to commercial sterility? Perhaps not, as Ariahu et al. [40] observed that all of the $\mathrm{z}$-values for clostridium sporogenes occurred at lower temperatures than thiamine destruction. Their data suggests that the microbiological safety might be attained at a tenfold lower temperature then thiamine degraded. But this is a very limited sampling and more research would help to understand if another (nutritional) safety variable might benefit our current model for commercial sterility.

Other factors that influence thiamine degradation during processing include blanching, changes in $\mathrm{pH}$, batter moisture, retort time, and retort temperature [41-43]. Thiamine exists in several ester forms, but depending on the food matrix, it can be degraded into several different products. For example, Dwivedi, Wantanabe and Ashai [44] performed an experiment with thiamine under alkaline conditions using paper chromatography to isolate the degradation of the molecule. They observed that thiamine was converted into thiochrome (a carbine form of thiamine), thiamine disulfide, and two derivatives of pyrimidine. In the same paper the researchers speculated that the activation of energy for the degradation of the thiazole ring occurs at a lower $\mathrm{pH}$ than the cleavage of the methylene bridge. The more acidic the food matrix, the more rapidly the thiazole group on the thiamine molecule will be cleaved and the methylene bridge destroyed. Differences also exist in thiamine levels retained at given $\mathrm{pH}$ treatments due to changes in time and temperature. For example, Rekha, et al. evaluated $\mathrm{pH}$, cooking time, and temperature to model the kinetics of thiamine degradation using a split red gram (Cajanus cajan L.) model that showed the greatest losses occurred at processing conditions most similar to those used to produce commercial canned cat food (pH 6.5, 15 minutes, and $120^{\circ} \mathrm{C}$ ) [41]. In a study with canned salmon Durance, et al. [36] showed that thiamine loss was lower when the processing time decreased fifteen percent. The $\mathrm{pH}$ may also influence the results as thiamine stability was greatest between $\mathrm{pH} 2.0$ and 4.0 chromatography to isolate the degradation of the molecule [45]. This suggests that as $\mathrm{pH}$ increases to a more neutral or alkaline $\mathrm{pH}$, thiamine will slowly become unstable and may degrade more easily in a pet food matrix.

Once thiamine has been broken down, there are several byproducts. These include hydrogen sulfite, elemental sulfur, 4-methyl-5-( $\beta$-hydroxyethyl) thiazole, and other minor products [34]. Thiamine is degraded by breaking the $\mathrm{CH}$ bond separating the pyrimidine and thiazole moieties leading to the destruction of the thiazole ring resulting in hydrogen sulfide production (1973). Morfee and Liska [46,47] conducted a study by incorporating thiamine in milk at $121^{\circ} \mathrm{C}$ for 50 minutes and observed sulfur as a major end product in more basic conditions. The thiamine-35 $\mathrm{S}$ (sulfur containing degraded thiamine) interacted with the protein in a milk model system and in evaporated milk. When the thiamine interacted with a combination of protein sources like lactose and sodium casein ate, it had the highest level of thiamine degradation as the $\mathrm{pH}$ shifted from 7.4 to 6.5 . Thiamine broke down the least (12.7 and $16.7 \%$, respectively) in model systems with high milk fat levels. Morfee and Liska [46] believed that the fat containing systems protect the thiamine. Processing conditions prior to retort preparation of a canned cat food was evaluated by Trible [42] and it was determined that batter moisture affected thiamine content at $65 \%$. As the retort time increased, thiamine retention decreased.

\section{Storage}

Commercial pet food is stored in a warehouse, on the store shelf, and in the pet owner's home for an extended period of time - for a canned pet food this can exceed two years. As the product ages, thiamine and other labile nutrients degrade. This happens starting at the very moment of harvest. For example, Poel, et al. [48] assessed the thiamine concentration in skeletal pork muscles during the harvesting process and showed that the concentration of thiamine decreased immediately upon harvest. This suggests that thiamine degradation occurs very quickly. But they also noted that total thiamine concentration increased in samples 96 and 216 hours after harvest. Perhaps as a result of thiamine phosphate ester production from the pig skeletal muscle during rigor. These thiamine phosphate esters include thiamine monophosphate, thiamine diphosphate, thiamine triphosphate, and thiamine tetra phosphate [48]. Peñas, et al. [49] stored four different dehydrated vegetables (dehydrated garlic, onions, potatoes, and carrots) in conditions comparable to a 
product sitting on a store shelf at three-month intervals for one year, and analyzed them for the concentration of various vitamins. In general, thiamine decreased in all four vegetables over time degrading more the longer it was stored [49]. The same situation may occur in pet food which has been stored for long periods. But supporting research is lacking.

\section{Food Preservatives}

Recent research has shown that various forms of sulfur dioxide, which were once used as a preservative to reduce odor of the meat in pet food has a negative impact on thiamine retention [50]. Sulfites were used to delay the reduction of myoglobin by inhibiting bacterial growth in meat [51-54]. By slowing the conversion of myoglobin into metmyoglobin, odor in meat containing foods is reduced and the red color preserved longer [52]. Thus, sulfur dioxide can increase the shelf life and lengthen the palatability of cooked meat. Several countries including the United States have banned sulfites in meats. Researchers investigated the kinetics of the thiamine degradation in order to minimize thiamine loss and (or) at least better predict and compensate for losses. It was determined that sulfites cleave the methylene bridge between the pyrimidine and thiazole moiety of the thiamine molecule. Both the sulfur ion and methylene bridge lead to thiamine instability during thermal processing.

Other ingredients in the food matrix may contain preservatives like sulfites and sulfur dioxide. These are inorganic bases found in dehydrated potatoes, potato flakes, wine, dried apples, corn gluten meal, and flavorings. These may unwittingly be added to pet foods and cause negative effects on thiamine in the can. Sulfur dioxide can also be used on fruits and vegetables to improve color retention [44]. Sulfur dioxide destroys thiamine by converting it into a thiazole base and pyridyl methanol sulfuric acid $[44,45,52]$. Even if a food contains an adequate amount of thiamine, it can be inactivated after coming into contact with sulfite treated meat because the sulfites change the structure of thiamine. Of course the destruction of thiamine in food depends on the dose of sulfur dioxide in the product [52]. For example, thiamine level decreased by $55 \%$ with $400 \mathrm{mg}$ sulfur dioxide and $95 \%$ with 1,000 $\mathrm{mg} / \mathrm{kg}$ sulfur dioxide in cat food [55]. This type of reduction is direct and predictable.
Another sulfur containing preservative commonly encountered in food systems is sodium bisulfite. In a simplified model, sodium bisulfite ( $0-1 \%$ levels) added to rice during the soaking step, reduced thiamine concentration $(0.08 \mathrm{~g} / 100 \mathrm{~g})$ of the finished product to undetectable levels $(<0.01 \mathrm{mg} / 100 \mathrm{~g}$; Vanier et al. [56]. Not to be confused with sodium bisulfate which is an acidifier and was shown to not directly affect thiamine concentrations any different than hydrochloric acid in a canned cat food application [57].

\section{Ingredients and their Relationship with Thiamine}

Canned pet foods are produced with many types of ingredients, including: water, meats, fats, starches gums, gels, vitamins, and minerals. Each of these may interact with thiamine differently and contribute to its degradation. In canned food, meat and fish play a prominent role in the recipe. One type of protein in particular that affects thiamine retention in pet food is fish. Fish and Shellfish contain a thiamine degrading enzyme, thiaminase. Hilker, et al. [8] observed that thiaminase tended to be higher in freshwater fish than oceanic fish. Thiaminase is commonly found in high concentration in the kidneys of carp, striped mullet, and alewives [58]. High concentrations of thiaminase were also found in the liver and intestines of Ukrainian fish [58]. The thiaminase enzyme degrades thiamine by a base-exchange reaction of the methylene group and pyridine moiety [58] (Figure 2). Thiaminase I comes from fish, shellfish, ferns, and some bacteria, and thiaminase II is found in some bacteria. Not only are these thiamine degrading enzymes derived from different sources, they hydrolyze the thiamine by different mechanisms. For example, thiaminase I requires a co-substrate in order to act as a transferase; whereas, thiaminase II hydrolyzes thiamine directly [59]. Fortunately, the thiaminase activity (because it is a protein) can be reduced with denaturing heat treatment [58]. In an extreme situation, the destruction of dietary thiamine could result from eating fish if the quantity of thiaminase was sufficient [58]. The processing of fish could reduce thiaminase activity in the final product [60]. Rendered fish meal used in pet food is extensively heated which should effectively inactivate thiaminase [53]. Thiaminase I can also be found in some bacteria such as P. thiaminolyticus and C. sporogenes [61].

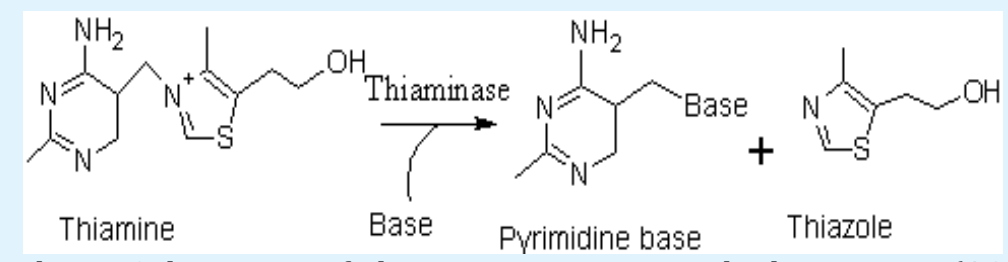

Figure 2: Chemical Base Substitution of Thiamine interacting with Thiaminase I (ACD/ChemSketch). 


\section{Open Access Journal of Veterinary Science \& Research}

Thiaminase is typically measured either by spectro fluoro metricthiochrome assay [62] or by radiochemical method with 14C-thiazole [63]. The radiochemical method uses thiamine hydrochloride in the $12 \mathrm{C}$ thiamine form and thiazeole-2-14C-thiamine hydrochloride (14C-thiamine) to measure the $14 \mathrm{C}$ thiazole activity [58].

\section{Other Factors}

Athar, et al. [64] evaluated vitamin retention in three different cereal grain mixtures that have native sources of b-vitamins(oats, maize, and a maize and pea mixture) and found thiamine retention differed such that the maize and pea mixture retained more thiamine than maize and (or) oats alone. The authors speculated that the oats should have had a greater retention because of the high oil content in the oats compared to the other grain ingredients. The authors had hypothesized that the higher oil content would alter the processing [64]. Athar, et al. concluded from the experiment that thiamine is more heat labile during extrusion compared to thiamine in the maize sample. This different response would suggest the possibility that we could select ingredients that promote greater thiamine retention and thereby minimize processing losses to some degree.

Szymandera-Buszka, et al. [23] evaluated other preservatives like rosemary extract using minced chicken as the model system in a thermal process. When added to minced chicken, rosemary extract extended the half-life of thiamine longer than casein hydrolysate. After the shelf life study, Szymandera-Buszka, et al. [23] concluded that the low oxidized fat and minced chicken with rosemary extract may have limited thiamine losses during storage. The authors also observed that samples with low oxidized fat and rosemary extract also had a longer shelf life. Rosemary may be used as a possible antioxidant source that could protect thiamine in canned pet food but more research would need to be conducted by incorporating meat into a pet food matrix as the model system. Then the rosemary and thiamine infused pet food matrix would need to be thermally processed in a retort at $121^{\circ} \mathrm{C}, 21$ PSI for 1 hour to represent typical canning conditions in the pet food industry.

One study evaluated the relationship between storage of pet food and nutrient retention over time. Mooney [65] conducted a shelf life study with extruded pet food comparing ambient storage $\left(20^{\circ} \mathrm{C}\right.$ and $50 \%$ $\mathrm{RH})$ conditions to pet food storage with high temperature and high humidity $\left(50^{\circ} \mathrm{C}\right.$ and $\left.75 \% \mathrm{RH}\right)$. Results from this study concluded that pet food stored in high humidity and high temperatures had a $24 \%$ thiamine loss after two weeks and 35\% thiamine loss after six weeks. When looking at the data from ambient storage, thiamine was relatively stable for the first three months but dropped to $65 \%$ of starting values by six months.

\section{Analytical Methods}

There are many methods to determine thiamine retention in food. However, two common techniques are based on fluorescence and high performance liquid chromatography. In order for thiamine to be measured in a given food sample, it must be extracted from the food matrix first. This is typically done using acid hydrolysis with low strength hydrochloric acid followed by enzyme digestion of starch using clara-diastase [66]. Potassium ferricyanide is a derivatizing agent that transforms thiamine into a thiochrome. Potassium ferricyanide oxidizes thiamine and its phosphate esters into thiochrome under alkaline conditions. Once thiamine has been converted to thiochrome, the sample can be refined through a gradient elution column and measured using fluorescence detection on the HPLC [66]. The HPLC is a rapid method that measures compounds using emission and excitation of wavelength [66]. Due to the sensitivity of thiamine to light, samples must be prepared in amber glassware to prevent any possible degradation before analysis.

Not only can the HPLC be used on thiamine in pet food, it can also be used to measure thiamine in the blood and urine of animals. Typically, thiamine and its phosphate esters are measured in the erythrocytes. Thiamine is carried in the erythrocytes or is bound to free plasma $[67,68]$.

Thiamine can also be measured by fluorescence with a fluorimeter [69]. In this procedure free thiamine is extracted from a $10 \mathrm{~g}$ sample in dilute acid after autoclaving. The solution is then incubated with buffered enzymes to release bound thiamine. After thiamine is oxidized to thiochrome, [17] the fluorometer measures the fluorescence of the standard solution with an input filter at $365 \mathrm{~nm}$ and output filter at $435 \mathrm{~nm}$. Then the standard solution fluorescence is compared it to the fluorescence of the thiamine extracted sample. Wherein, the intensity of the fluorescence increases with increasing concentration of the thiamine in the sample. This method is sensitive and has specific quantification [17].

\section{Summary}

Even though thiamine deficiencies are a well-known problem in the pet food industry, most of the survival research has been conducted using human food matrices. Pet food differs from human "foods" as they 


\section{Open Access Journal of Veterinary Science \& Research}

are complete diets containing all the nutrients the animal requires. This creates interactions among the various elements and the deficiencies are more serious as no other food item will offset the insufficiency. This shortcoming for information in canned pet food products should be corrected.

A time and temperature relationship affects the concentration of thiamine during thermal processing as thiamine is heat labile. However, more research needs to be conducted on the thermal kinetic relationship of time and temperature during thermal processing of canned pet foods containing thiamine. Furthermore, indepth research also needs to be conducted to consider other processing factors, such as $\mathrm{pH}$ and ingredient impact of thiamine in pet food products.

Research has shown that using ingredients with lowoxidized fats and using preservatives like rosemary extract may limit thiamine losses during thermal processing [23]. Sulfites that are used in food as preservatives also have a negative impact on thiamine and should be used as little as possible in pet food products [44]. Enzymes such as thiaminase will inactivate thiamine in canned pet food. When developing pet foods, the industry should be conscious when determining what ingredients are added to the food matrix in order to reduce thiamine loss. Overall, time, temperature, and ingredient interactions reduce thiamine concentration during thermal processing of pet food because thiamine is heat labile.

\section{References}

1. Jansen BCT, Donath WF (1926) On the isolation of the anti-beriberi vitamin. Proc $\mathrm{K}$ Acad Wet Amsterdam 29: 1390.

2. Rumbeiha WK, Morrison J (2010) A review of class I and class II pet food recalls involving chemical contaminants from 1996 to 2008. American College of Toxicology 7(1): 60-66.

3. National Research Council (2006) Nutrient requirements of dogs and cats. Washington, D.C.: Washington DC: National Academies Press.

4. Bischoff K, Rumbeiha WK (2012) Pet food recalls and pet food contaminants in small animals. Vet Clinical Small Animal 42: 237-250.

5. Dreyfus PM, Victor M (1961) Effects of thiamine deficiency on the central nervous system. Am J Clin Nutr 9: 414-425.

6. Moon S, Kang M, Park H (2013) Clinical signs, MRI features, and outcomes of two cats with thiamine deficiency secondary to diet change. Journal of veterinary science 14(4): 499-502.

7. Davidson MG (1992) Thiamin deficiency in a colony of cats. Vet Rec 130(5): 94-97.

8. Hilker DM, Peter OF, (1966) Anti- thiamine activity in Hawaii fish. Journal of Nutrition 89(4): 419-421.

9. Chang Y, Chiu P, Lin C, Liu I, Liu C (2016) Outbreak of thiamine deficiency in cats associated with the feeding of defective dry food. Journal of Feline Medicine and Surgery 1-8.

10. Singh M, Thompson M, Sullivan N, Child G (2005) Thiamine deficiency in dogs due to the feeding of sulphite preserved meat. Australian Veterinary Journal 83(7): 412-417.

11. Irle E, Markowitsch HJ (1982) Thiamine deficiency in the cat leads to severe learning deficits and widespread neuroanatomical damage. Experimental Brain Research 48(2): 199-208.

12. Anholt H, Himsworth C, Britton A (2016) Polioencephalomalacia and heart failure secondary to presumptive thiamine deficiency, hepatic lipidosis, and starvation in 2 abandoned Siamese cats. Vet Pathol 53 (4): 840-843.

13. Palus VJ, Penderis S, Jakovlijevic, Cherubini GB (2010) Thiamine deficiency in a cat: resolution of MRI abnormalities following thiamine supplementation. 12(10): 807-810.

14. Fattal-Valevski A (2011) Thiamine (vitamin B1). Complementary Health Practice Review 16(1): 1220.

15. Gregory J (1997) Bioavailability of thiamin. European Journal of Clinical Nutrition 51: S34-S37.

16. Bubber P, Ke Z, Gibson GE (2004) Tricarboxylic acid cycle enzymes following thiamine deficiency. Neurochemistry International 45(7): 1021-1028.

17. Manzetti S, Zhang J, van der Spoel D (2014) Thiamin function, metabolism, uptake, and transport. Biochemistry 53(5): 821-835.

18. Chabrie 're E, Charon MH, Volbeda A, Pieulle L, Hatchikian EC, et al. (1999) Crystal Structures of the key anaerobic enzyme pyruvate: ferredoxin oxidoreductase, free and in complex with pyruvate. Nat Struct Mol Biol 6(2): 182-190.

19. Butterworth RF 2006. Thiamin. In: Shils ME, Shike M, Ross AC (Eds.), Modern nutrition in health and 


\section{Open Access Journal of Veterinary Science \& Research}

disease. 10th ed. Lippincott Williams \& Wilkins, Baltimore, MD pp. 426-433.

20. Gibson Ge, Blass JP (2007) Thiamine- dependent processes and treatment strategies in neuro degeneration. 9(10): 1605-1619.

21. Ellefson WC (1985) Thiamin. In: J Augustin (Eds.) Methods of vitamin assay 4th (Edn.) Wiley, New York, 349-363.

22. Williams RR, Cline JK (1936) Synthesis of vitamin B1. J Am Chem Soc 58(8): 1504-1505.

23. Szymandera-Buszka K, Hes M, Waszkowiak K, Jedrusek-Golin'Ska A (2014) Thiamine losses during storage of pasteurised and sterilized model systems of minced chicken meat with addition of fresh and oxidized fat, and antioxidants. Acta Scientiarum Polonorum, Technologia Alimentaria 13(4): 393-401.

24. Jun L, EL Frank (2008) Rapid HPLC measurement of thiamine and its phosphate esters in whole blood (automation and analytical techniques). Clinical Chemistry 54(5): 901.

25. Kraut J, Reed HJ (1962) The crystal structure of thiamine hydrochloride (vitamin b1). Acta Cryst 15: 747-757.

26. Davis RE, Icke GC (1983) Clinical chemistry of thiamin. Advances in Clinical Chemistry 23: 93-140.

27. Matsukawa TH, Hirano, Yurugi S (1970) Preparation of thiamine derivatives and analogs. Methods in Enzymology 18: 141-162.

28. (2011) Scientific opinion on the safety and efficacy of vitamin B1 (thiamine mononitrate and thiamine hydrochloride) as a feed additive for all animal species based on a dossier submitted by DSM nutritional products. EFSA Journal 9(11): 2413.

29. Farrer KTH (1945) The thermal destruction of vitamin B1. Biochemical Journal 41(2): 167-169.

30. AAFCO (2014) Official publication of the Association American Feed Control Officials.

31. Berry MR, Pflug IJ (1993) Canning principles. Encyclopedia of food science and nutrition, tenvolume set 816-824.

32. Hendriks WH, Emmens MM, Trass B, Pluske JR (1999) Heat processing changes the protein quality of canned cat foods as measured with rat bioassay. Journal of Animal Science 77(3): 669-676.
33. Awuah GB, Ramaswamy HS, and Economides A (2006) Thermal processing and quality: Principles and overview. Chemical Engineering \& Processing: Process Intensification 46(6): 584-602.

34. Balsa-Canto E, Fernandez MR, Banga JR (2007) Optimal design of dynamic experiments for improved estimation of kinetic parameters of thermal degradation. Journal of Food Engineering 82(2): 178-188.

35. Zajko AP, Klimant I (2013) The effects of different sterilization procedures on the optical polymer oxygen sensors. Sensors and Actuators B 177: 8693.

36. Durance TD (1997) Improving canned food quality with variable retort temperature processes. Trends in Food Science \& Technology 8(4): 113-118.

37. Fellows P (2009) Food processing technology principles and practice. $3^{\text {rd }}$ (Edn.), Woodhead Publishing, Oxford, pp. 1-928.

38. Goff D, Hartel R (2013) Thermal destruction of microorganisms $7^{\text {th }}$ (Edn.), Springer, Canada.

39. Pflug J, Christensen R (1980) Converting an f-value determine on the basis of one $\mathrm{z}$-value to an f-value determined on the basis of a second $\mathrm{z}$-value. J Food Sci 45(1): 35-40.

40. Ariahu C, Ogunsua A (2000) Thermal degradation kinetics of thiamine in periwinkle based formulated low acidity foods. International Journal of Food Science and Technology 35(3): 315-321.

41. Rekha P, Nisha S, Singhal, Aniruddha B Pandit (2004) A Study on Degradation Kinetics of Thiamine in Red Gram Splits (Cajanus Cajan L.). Food Chemistry 85 (2004): 591-598.

42. Trible SD (2016) The effects of canning on Bvitamin retention in a model cat diet with an emphasis on thiamine. Manhattan KS: Kansas State University.

43. Słupski J (2012) Effect of freezing and canning on the thiamine and riboflavin content in immature seeds of five cultivars of common bean (phaseolus vulgaris L.). International Journal of Refrigeration 35(4): 890-896.

44. Dwivedi BK, Arnold RG (1973) Chemistry of thiamine degradation in food products and model systems: A review. Journal of Agriculture of Food Chemistry 21(1): 54-59. 


\section{Open Access Journal of Veterinary Science \& Research}

45. Trang HC, Chen F, Haley-Zitlin V, Young K (2013) Development of HPLC methods for the determination of water-soluble vitamins in pharmaceuticals and fortified food products. ProQuest Dissertations and Theses.

46. Morfee TD, Liska BJ (1971) Distribution of thiamine degradation products in simulated milk systems. Journal of Dairy Science 54(7): 1082-1086.

47. Morfee TD, Liska BJ (1972) Elemental sulfur: Degradation product of thiamine in model Systems1. Journal of Dairy Science 55(1): 123-125.

48. Poel C, Bäckermann S, Ternes W (2009) Degradation and conversion of thiamin and thiamin phosphate esters in fresh stored pork and in raw sausages. Meat Science 83(3): 506-510.

49. Peñas E, Sidro B, Ullate M, Vidal-Valverde C, Frias J (2013) Impact of storage under ambient conditions on the vitamin content of dehydrated vegetables. Food Science and Technology International 19(2): 133-141.

50. Malik R, Sibraa D (2005) Thiamine deficiency due to Sulphur dioxide preservative in 'pet meat'-a case of déjà vu. Australian veterinary journal 83: 408-411.

51. Clydesdale FM, Ho CT, Lee CY, Mondy NI, Shewfelt RL (1991) The effects of postharvest treatment and chemical interactions on the bioavailability of ascorbic acid, thiamin, vitamin A, carotenoids, and minerals. Critical Reviews in Food Science and Nutrition 30(6): 599-638.

52. Singh M, Thompson M, Sullivan N, Child G (2005) Thiamine deficiency in dogs due to the feeding of sulphite preserved meat. Australian Veterinary Journal 83(7): 412-417.

53. Markovich JE, Heinze CR, Freeman LM (2013) Thiamine deficiency in dogs and cats. Journal of the American Veterinary Medical Association 243(5): 649-656.

54. Steel RJS (1997) Thiamine deficiency in a cat associated with the preservation of 'pet meat with sulphur dioxide. Australian Veterinary Journal 75(10): 719-721.

55. Studdert VP, Labuc RH (1991) Thiamine deficiency in cats and dogs associated with feeding of sulfite preserved meat. Aust Vet J 68(2): 54-57.

56. Vanier NL, Paraginski RT, Berrios De JJ, Conceição Oliveira L Da, Cardoso Elias M (2015) Thiamine content and technological quality properties of parboiled rice treated with sodium bisulfite: Benefits and food safety risk. Journal of Food Composition and Analysis 41: 98-103.

57. Trible SD (2017) The Effect of Processing and Ingredient Interactions on Thiamine Degradation in Canned Cat Food: A Modern Nutrition-Health Dilemma. J Vet Sci Res 2(2): 000130.

58. Zajicek J, Tillitt D, Honeyfield D, Brown S, Fitzsimons J, et al. (2005) A method for measuring total thiaminase activity in fish tissues. Journal of Aquatic Animal Health 17(1): 82-94.

59. Edwin EE (1979) Determination of thiaminase activity using thiazole-labeled thiamine. Methods in Enzymology 62: 113-117.

60. Sivasankar B (2004) Thiaminase. In Food processing and preservation. $6^{\text {th }}$ (Edn.) PHI Learning Limited, New Delhi.

61. Kraft CE, Gordon ER, Angert ER (2014) A rapid method for assaying thiaminase I activity in diverse biological samples. Plos One 9(3): e92688.

62. Fujita A (1954) Thiaminase. Advances in Enzymology 15: 389-421.

63. Edwin EE, Jackman R (1974) A rapid radioactive method for determination of thiaminase activity and its use in the diagnosis of cerebrocortical necrosis in sheep and cattle. Journal of the Science of Food and Agriculture 25(4): 357-368.

64. Athar N, Hardacre A, Taylor G, Clark S, Harding R (2006) Vitamin retention in extruded food products. Journal of Food Composition and Analysis 19(4): 379-383.

65. Mooney A (2016) Stability of essential nutrients in pet food manufacturing and storage. Manhattan, Kan Manhattan, Kan: Kansas State University.

66. Analytical Methods Committee (2000) Determination of thiamine and riboflavin in pet foods and animal feeding stuffs. The Royal Society of Chemistry 125: 353-360.

67. Marks SL, Lipsitz D, Vernau KM, Dickinson PJ, Draper W (2011) Reversible encephalopathy secondary to thiamine deficiency in 3 cats ingesting commercial diets. Journal of Veterinary Internal Medicine 25(4): 949-953.

68. Talwar D, Davidson H, Cooney J, O'reilly D (2000) Vitamin B-I status assessed by direct measurement of thiamin pyrophosphate in erythrocytes or whole 
blood by HPLC: Comparison with erythrocyte transketolase activation assay. Clin Chem 46(5): 704-710.
69. AOAC official method 942.23 (2012) Thiamine (vitamin B1) in human and pet foods. AOAC 11-13. 